\title{
EXPERIMENTAL VALIDATION OF CUMULATIVE SURFACE LOCATION ERROR FOR TURNING PROCESSES
}

\author{
AdAm K. KisS*, DANiEl Bachrathy \\ Budapest University of Technology and Economics, Department of Applied Mechanics, 1111 Budapest, \\ Muegyetem rkp. 5., Hungary \\ * corresponding author: kiss_a@mm.bme.hu
}

\begin{abstract}
The aim of this study is to create a mechanical model which is suitable to investigate the surface quality in turning processes, based on the Cumulative Surface Location Error (CSLE), which describes the series of the consecutive Surface Location Errors (SLE) in roughing operations. In the established model, the investigated CSLE depends on the currently and the previously resulted SLE by means of the variation of the width of cut. The phenomenon of the system can be described as an implicit discrete map. The stationary Surface Location Error and its bifurcations were analysed and flip-type bifurcation was observed for CSLE. Experimental verification of the theoretical results was carried out.
\end{abstract}

KEYWORDS: surface quality, Surface Location Error, implicit map, flip bifurcation.

\section{INTRODUCTION}

In the industry for production and manufacturing, turning is a widely used method. However, high material removal rate often cannot be accomplished in practice due to the instability of the cutting process. This machining process induces harmful vibrations which are responsible for unacceptable surface quality [1. The phenomenon is the self-excited vibration, the so-called chatter vibration which comes from loss of stability of the periodic vibration due to the surface regeneration effect [2]. This effect can be modelled with delayed differential equations $(D D E)[3$.

The so-called stability chart [4 presents the chatterfree (stable) parameter domain which is usually illustrated along the parameters of the spindle speed and the depth of cut, calculated by numerical methods [5, 6]. The most productive parameter regions are located between the pockets of the the so-called lobes [7], where large cutting force can occur due to the high depth of cut ratio which leads substantial Surface Location Error (SLE).

The stability chart is usually used at the roughing processes. Due to the fact, that SLE is relevant at finishing operations [8], its effect is usually neglected in the model of roughing operations, but despite this, it can has significant impact on the surface quality in case of consecutive immersions - which is typical for roughing operation.

We introduce a new type of surface error calculation which considers the effect of the series of SLEs during several consecutive immersion.

The oversize of a workpiece is removed with several consecutive immersions in roughing machining process. At every immersion, the machined surface differs from the desired one due to the SLE. The actual offset error $S L E_{\mathrm{i}}$ modifies the subsequent immersion. This modified immersion generates different cutting force

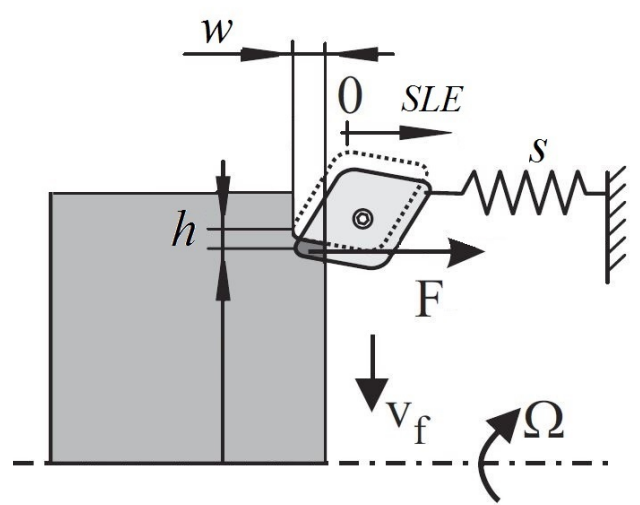

FiguRE 1. SDoF turning dynamical model.

which leads another, modified offset error $S L E_{\mathrm{i}+1}$. During this process, the SLE can be accumulated and leads a new surface quality parameter denoted by the Cumulative Surface Location Error (CSLE) [9, 10.

The evaluation of the series of SLEs values and its stability problems are investigated in turning operations on a Single-Degree-of-Freedom (SDoF) mechanical model.

\section{Evolution of Cumulative Surface LOCATION ERROR}

The constructed mechanical model and the main steps of the SLE computation are summarised as follows.

\subsection{Mechanical Model}

In the mechanical model, the workpiece is considered as a rigid body and the cutting tool is considered as Single-Degree-of-Freedom ( $S D o F)$ model, as shown in Figure 1 In the figure, some technological parameters - which correspond to the material removal rate - and 


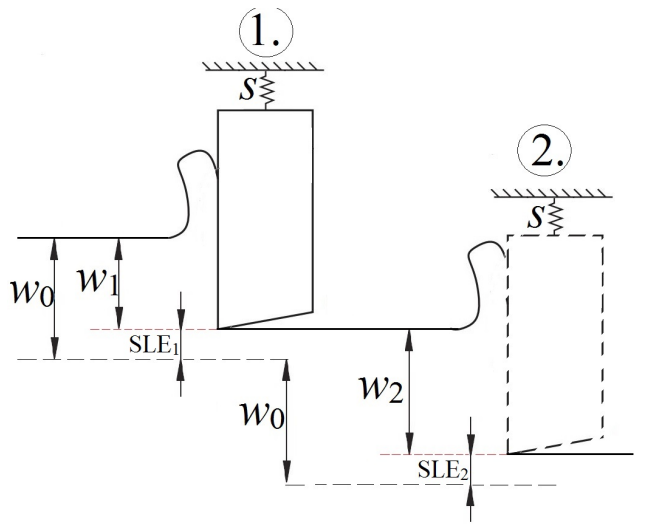

Figure 2. Evolution of the Surface Location Error $(S L E)$ in consecutive immersions.

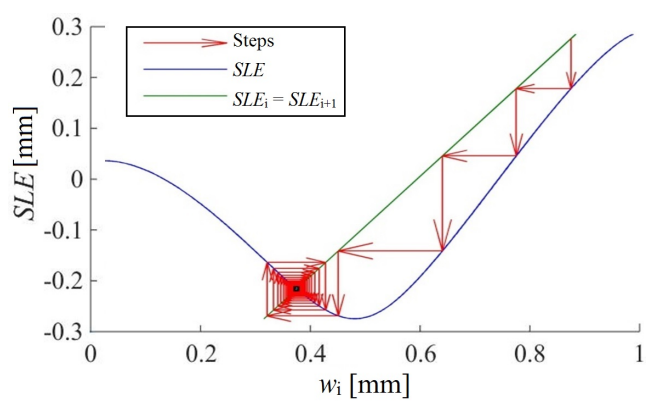

FIGURE 3. Schematic representation of map.

the main mechanical parameters are represented. $W$ is the width of the chip, $h$ is the chip thickness or the feed per revolution, $v_{f}$ is the feed movement, $\Omega$ is the spindle speed, $F$ is the cutting force generated from the material removal process, $s$ is the stiffness of the tool-holder and SLE is the Surface Location Error which is the distance between the machined and desired surface. The contour of the surface is defined by the position of the turning insert. In other words, the $S L E$ is the static displacement error of the turning insert which is generated by the $F$ constant cutting force.

In this model, the so-called surface regeneration effect of the machining process is not considered. This effect would lead to time dependent cutting force function, which may result stability problems, described in details in [11. In this study, only the static displacement is analysed, hence exclusively the stationer component of the chip thickness is used.

Note, that in case of the surface regenerative models, the tool is considered to be flexible in the direction of the feed velocity [12], but, in our case it is flexible perpendiculat to the feed velocity.

\subsection{Calculation of CSLE}

The model considers the evolution of the width of the chip as follows: in roughing operation the desired width of cut $w_{0}$ is modified by the actual $S L E_{\mathrm{i}}$ and the subsequent $S L E_{\mathrm{i}+1}$ (see Figure 2):

$$
w_{\mathrm{i}+1}\left(S L E_{\mathrm{i}}, S L E_{\mathrm{i}+1}\right)=w_{0}+S L E_{\mathrm{i}}-S L E_{\mathrm{i}+1} .
$$

In the stationary position the spring force is equal to the cutting force:

$$
S \cdot S L E_{\mathrm{i}+1}=F_{\mathrm{i}+1},
$$

so the subsequent surface error can be calculated as:

$$
S L E_{\mathrm{i}+1}=\frac{1}{s} F\left(W_{\mathrm{i}+1}\left(S L E_{\mathrm{i}}, S L E_{\mathrm{i}+1}\right)\right) .
$$

The above implicit discrete map describes how one $S L E$ develops into another SLE over of a subsequent immersion. The $S L E$ values follow each other one by one. These series of the SLEs can converge to a fix point (see Figure 3; where 45 degree line represents points where $S L E_{\mathrm{i}}=S L E_{\mathrm{i}+1}$ ) which is denoted by CSLE.

This solution is obtained by two different methods. One of them is a numeric iteration of the map and the other one is a numerically calculated roots of the analytical function defined by Eq.(3), made by Multi-Dimensional Bisection Method [13].

The characteristic of the map is highly dependent on the applied cutting force model. Some typical approximations of the cutting force as the linear, the threequarter rule [14] and the cubic polinomial rule [15] were investigated.

\subsection{Stability Analysis of CSLE}

The stability of the investigated system is determined based on the derivative of Eq. 3 with respect to $w$ width of cut at the fix points [16]. Fold type bifurcation occurs, when the derivative is greater than 1 , which leads to stabiliy loss of the CSLE and also could lead to bistable region. Another case, when the derivative is smaller than -1 , this situation corresponds to the period doubling or flip-type bifurcation.

After some mathematical transformation, it can be proved that only the flip-type bifurcation can occure for this model. It happens when the derivative is equals to $-s / 2$. For a realistic environment, the $s$ stiffness is positive and the derivative of the turning force function is also positive for the usual turning force models, therefore the mechanical model is stable. But, there can exist some special cases, when the cutting force in the function of the width of cut turn out to negative sign (and its derivative is also negative) in real machining environment, not only in mathematical sense. For instance, in our experimental measurement (see in details in the following subsection), the resultant cutting force was negative due to the edge geometry of the applied insert. Fold or saddle node bifurcation point cannot be obtained for physical meaningful parameters [10]. 


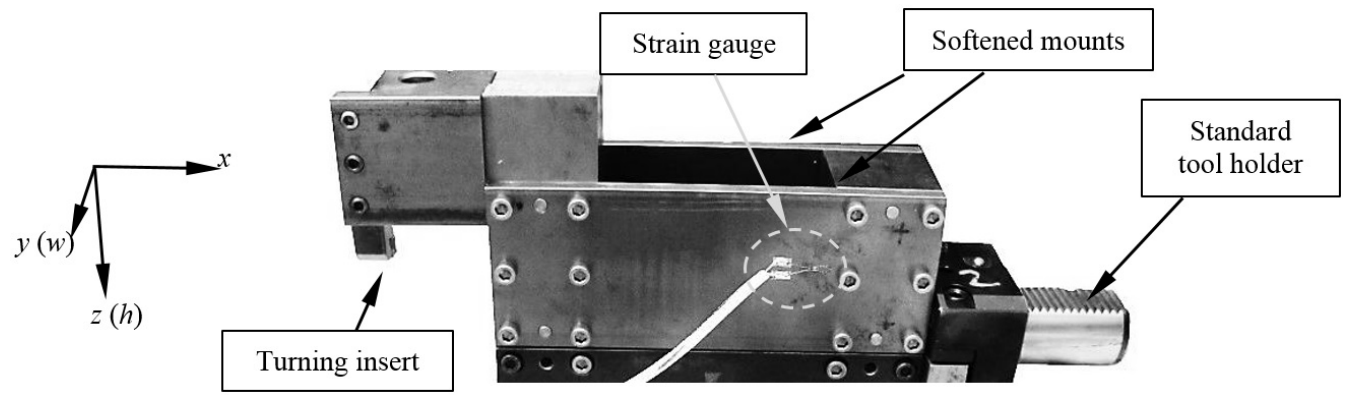

Figure 4 . The softened tool mount and the fabricated strain gauges.

\begin{tabular}{|c|c|c|c|c|}
\hline & $1^{s t}$ strain gauge & $2^{\text {nd }}$ strain gauge & $3^{r d}$ strain gauge & $4^{\text {th }}$ strain gauge \\
\hline \multicolumn{5}{|l|}{ Def. calib. coeff. } \\
\hline$\left[\frac{\text { deformation }}{\text { strain value }}\right]=\left[\frac{\mathrm{mm}}{\mu \mathrm{m} / \mathrm{m}}\right]$ & $-1.56 \cdot 10^{4}$ & $1.06 \cdot 10^{4}$ & $0.75 \cdot 10^{4}$ & $-0.57 \cdot 10^{4}$ \\
\hline \multicolumn{5}{|l|}{ Force calib. coeff. } \\
\hline$\left.\frac{\text { force }}{\text { strain value }}\right]=\left[\frac{\mathrm{N}}{\mu \mathrm{m} / \mathrm{m}}\right]$ & $-8.64 \cdot 10^{6}$ & $6.76 \cdot 10^{6}$ & $4.56 \cdot 10^{6}$ & $-3.38 \cdot 10^{6}$ \\
\hline \multicolumn{5}{|l|}{ Stiffness } \\
\hline$\left.\frac{\text { force calib. coeff. }}{\text { deformation calib. coeff. }}\right]=\left[\frac{\mathrm{N}}{\mathrm{mm}}\right]$ & 533.22 & 636.71 & 614.07 & 593.43 \\
\hline
\end{tabular}

Averaged stiffness $\left[\frac{\mathrm{N}}{\mathrm{mm}}\right]$

599.36

TABLE 1. Calibration coefficients and the calculated stiffness.

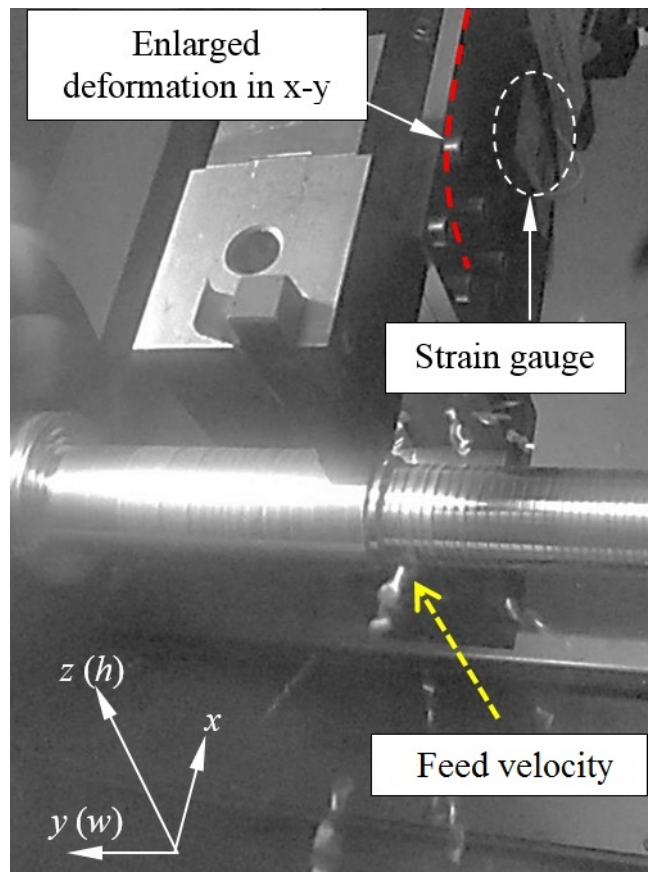

FiguRE 5. Experimental setup.

\section{Experimental VAlidation}

Experimental verification of the theoretical results was carried out in a 2 axes universal turning machine center. The development of the resulted offset error along the direction of the width of the chip (SLE) was investigated on a given workpiece during five consecutive immersions, as shown in Figure 5 For better measurement conditions, a softened tool holder

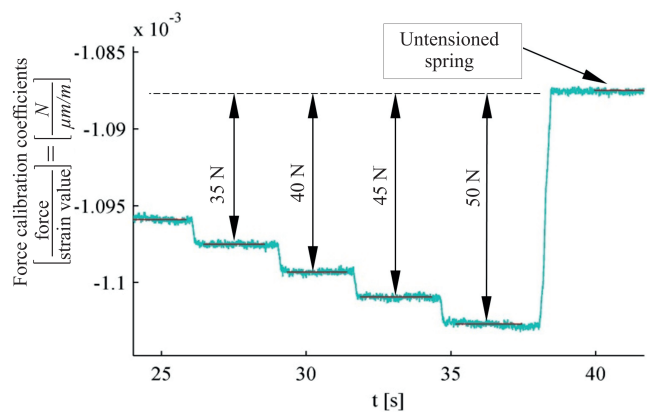

Figure 6. Measured sign (blue) and averaged values (red lines) of force calibration.

was used which results higher offset error. The tool holder is described in details in [17].

The generated cutting force and the deformation were measured by means of strain gauges installed on the softened tool holder (see Figure 4). According to the strain gauges, the measurement instrument had to be calibrated for force and for deformation measurement.

The force calibration was carried out as the artificial deformations and forces were generated meanwhile the signals from the strain gauges were recorded (see Figure 6). The calibration coefficients (presented in Table 1) were determined from the averaged measured data by applying linear regression by means of ordinary least squares approach. The calculated stiffness: $\mathrm{s} \cong 600 \mathrm{~N} / \mathrm{mm}$.

We carried out a series of measurements using different turning process parameters. In a single test, 5 consecutive cuts were performed with fixed cutting 


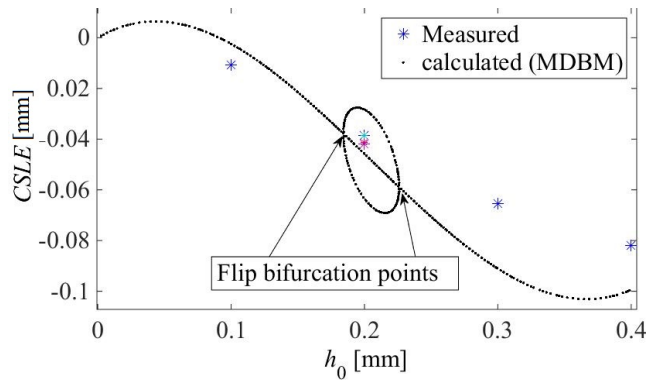

Figure 7. Calculated CSLE function and the measured displacements (blue stars denote the converged solutions; colored stars denote the period doubling characteristics).

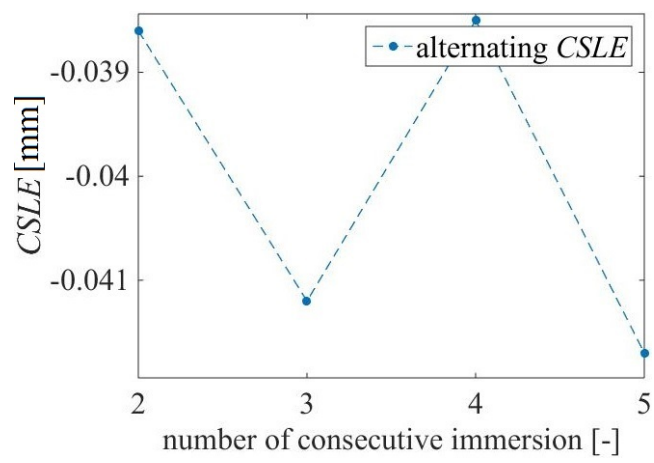

Figure 8. Measured period doubling characteristics; Parameters: $w=4[\mathrm{~mm}], h_{0}=0.2[\mathrm{~mm}]$.

parameters to investigate the development of SLE series. The measured cutting force was negative due to the applied insert. The reason of this phenomenon may come from geometrical setup, the edge geometry and the shape of the inserts. A fitting method was applied based on the measured averaged cutting forces to determine the specific cutting coefficients of the widely used polynomial cutting force function [15]. The material of the workpiece was AlMgSi0.5 aluminium alloy.

The CSLE is calculated numerically from Eq.(3) for the fitted specific cutting coefficients and it is compared to the measured values.

For the computed CSLE, flip-type bifurcation is occurred at certain parameter points at $h_{0}=0.17$ $[\mathrm{mm}]$ and $0.23[\mathrm{~mm}]$ (see Figure 7 ) where the stable solution becomes unstable and creates a periodic-2 solution. Between these parameter points, the series of the meeasured $S L E$ values do not converge to a certain value, but they alternate around 2 values (see Figure 87. However, the amplitude of the measured alternating (bifurcating) displacement error has much smaller amplitude than the calculated one. The source of this large error could be the small number of consecutive cuts in the measurements, while the theoretical amplitude refers to the steady state amplitude after an infinite number of cuts.

\section{Conclusions}

In the present study, we show a new type of stability problem, which can occur during roughing process. This CSLE-stability problem leads to an unpredictable final Surface Location Error, which can affect the finishing operation substantially. An experimental verification of the theoretical results was carried out in a softened machine tool environment, where a correlation was found between the measured and the computed values.

In most of the cases, the series of CSLE values of one roughing test converged to certain value, showing also good correlation with the computed CSLEs. In the predicted parameter range of period-2 solutions, the series of the measured deformations shows alternating characteristic. We can state, that the predicted phenomenon is validated by measurements.

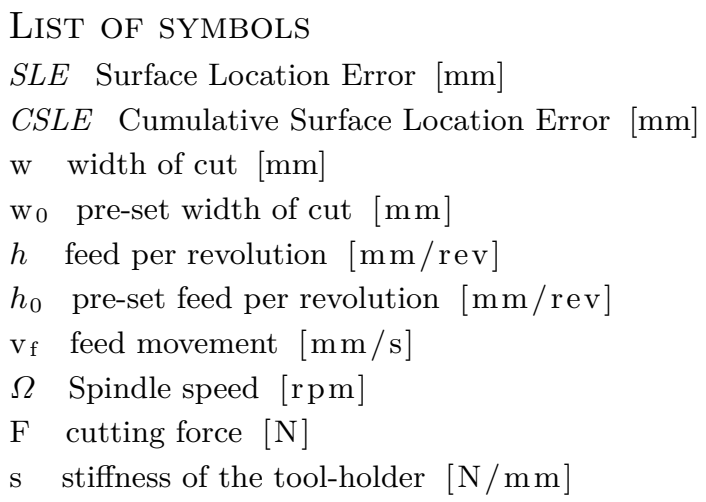

\section{ACKNOWLEDGEMENTS}

This paper was supported by the Hungarian Scientific Research Fund - OTKA PD-112983 and the Janos Bolyai Research Scholarship of the Hungarian Academy of Sciences.

\section{REFERENCES}

[1] S. A. Tobias. Machine tool vibration. Blackie and Son, Ltd, London 1965.

[2] J. Tobias, L. Spacek. Self-excited vibrations on machine tools, 1954.

[3] G. Stepan. Retarded dynamical systems: stability and characteristic functions. Longman Scientific and Technical 1989.

[4] Y. Altintas, E. Budak. Analytical prediction of stability lobes in milling. CIRP Annals - Manufacturing Technology 44(1):357-362, 1995.

[5] T. Insperger, G. Stepan. Semi-discretization for time-delay systems. Springer, Applied Mathematical Sciences 2011.

[6] D. Bachrathy, G. Stepan. Improved prediction of stability lobes with extended multi frequency solution. CIRP Annals - Manufacturing Technology 62(1):411-414, 1995.

[7] M. J. Reith, G. Stepan. Optimization of material removal rate for orthogonal cutting with vibration limits. Periodica Polytechnica-Mechanical Engineering 56(2):91-97, 2012. DOI:10.3311/pp.me.2012-2.03 
[8] W. A. Kline, R. E. DeVor, I. A. Shareef. The prediction of surface accuracy in end milling. ASME Journal of Engineering for Industry 104(3):272-278, 2009.

[9] A. Kiss, D. Bachrathy. Explicit model of cumulative surface location error for milling processes. 12th Hungarian Conference on Theoretical and Applied Mechanics 2015.

[10] A. Kiss. Cumulative surface location error for cutting processes. MSc thesis, BME, Department of Applied Mechanics 2014.

[11] R. Szalai, G. Stepan. Lobes and lenses in the stability chart of interrupted turning. Journal of Computational and Nonlinear Dynamics 1(3):205-212, 2006.

[12] T. Insperger, D. A. W. Barton, G. Stepan. Criticality of hopf bifurcation in state-dependent delay model of turning processes. International Journal of Non-Linear Mechanics 43(2):140-149, 2013. DOI:10.1016/j.ijnonlinmec.2007.11.002
[13] D. Bachrathy, G. Stepan. Bisection method in higher dimensions and the efficiency number. Periodica Polytechnica-Mechanical Engineering 56(2):81-86, 2012.

[14] O. Kienzle. Spezifische schnittkrafte bei der metallbearbeitung. Werkstattstechnik und Maschinenbau 47(1):224-225, 1957.

[15] H. Shi, S. Tobias. Theory of finite-amplitude machine-tool instability. International Journal of Machine Tools and Manufacture 24(21):45-69, 1984.

[16] J. T. Sandefur. Discrete dynamical systems : Theory and applications 1990.

[17] M. J. Reith, D. Bachrathy, G. Stepan. Stability properties and optimization of multi-cutter turning operations. ASME, International Design Engineering Technical Conferences and Computers and Information in Engineering Conference: DETC2013: Portland, USA, Oregon 2013. DOI:10.1115/detc2013-12347 